\title{
FINITE ELEMENT APPROXIMATIONS FOR THE STATIONARY LARGE EDDY SIMULATION MODEL
}

Abstract. Some approximation procedures are presented for the system of equations arising from the large eddy simulation of turbulent flows. Existence of solutions to the approximate problems is proved. Discrete solutions generate a strongly convergent subsequence whose limit is a weak solution of the original problem. To prove the convergence theorem we use Young measures and related tools. We do not limit ourselves to divergence-free functions and our results are in particular valid for finite element approximations where one usually does not use functions with divergence equal to zero.

1. Introduction. We will consider steady turbulent flows of fluids in a bounded set $\Omega$ in $\mathbb{R}^{d}\left(d=2\right.$ or 3 ). Equations for the velocity $v: \Omega \rightarrow \mathbb{R}^{d}$ and pressure $p: \Omega \rightarrow \mathbb{R}$ are given by

$$
\begin{aligned}
v \cdot \nabla v-\operatorname{div} A(y, D v)-\nu \Delta v+\nabla q & =f, \\
\operatorname{div} v & =0,
\end{aligned}
$$

with viscosity $\nu>0$ and the boundary condition

$$
v(x)=0 \quad \text { on } \partial \Omega,
$$

where $D v=\frac{1}{2}\left(\nabla v+\nabla v^{T}\right)$ denotes the symmetric part of the gradient and $y$ is defined by $y=(\widetilde{v}, D \widetilde{v}, \widetilde{v v}, \mid \widetilde{D v \mid D v})$. The tilde denotes convolution with some smooth filter function, namely $\tilde{v}(x)=\int_{\Omega} v(y) \varphi_{\delta}(x-y) d y$ (see Subsection 1.2 for the definition of the filter function). The nonlinear and nonlocal term $A$ (the so-called turbulent term) is given by

$$
A(y, D v)=c(y)|D v| D v
$$

2010 Mathematics Subject Classification: 76F65, 76M10, 35Q30.

Key words and phrases: large eddy simulation, Young measures, finite element method. 
with a function $c$ continuous with respect to all variables and satisfying the condition

$$
0<\alpha \leq c \leq \beta<\infty .
$$

The equations come from the Large Eddy Simulation (LES) of turbulent incompressible flows. The idea of this approach bases on decomposing the quantities which describe the flow (velocity, pressure, body forces) into two parts: one part containing the large flow structures (large scales) and the remainder containing the small scales. In LES, the large flow structures are defined by averaging the velocity, pressure, etc. in space. A usual way is to define the spatial average by convolving these quantities with an appropriate filter function with a filter width $\delta>0$. This filter function should filter out the small scale structures, of size smaller than $O(\delta)$. In LES only large flow structures are computed accurately.

This approach makes sense in applications. Usually, the behaviour of large eddies is important and more significant than all the small eddies. However, prediction of the behaviour of large eddies is not possible without taking into account the interaction coming from the small eddies.

The derivation of the equations for the large scales is now rather clear. First we convolve the Navier-Stokes equations with some function (filter). This leads to the so-called space-averaged Navier-Stokes equations. To obtain equations for the large scales quantities we add a constitutive relation modeling the contribution of small structures into the flow. Different types of modeling are studied; for details see [9] or [11]. In this paper we consider a slight generalization of the Germano model where the turbulent term is defined by (1.3) with assumptions (1.4). This leads to the equations (1.1). We refer to [7] for more details on the derivation of the model.

In [5] existence, convergence and error estimate for approximate solutions to the Smagorinsky-Ladyzhenskaya model were studied. One can consider this model as a simplification of the Germano model, namely the Germano model with constant and positive function $c$; see 9] for details. Unfortunately, the methods proposed are not sufficient when an additional nonlocal and nonlinear term, namely the dependence of the function $c$ on $y$, occurs.

In this paper we present and analyse some approximation procedures for (1.1). In contrast to [8] we do not limit ourselves to divergence-free function spaces. Our results are in particular valid for finite element approximations where one usually does not use functions with divergence equal to zero.

1.1. Numerical motivation. The ideal approach is to compute a numerical approximation of the solution of the Navier-Stokes equations directly by using some finite element approximations. This approach, called direct numerical simulation (DNS), tries to solve the Navier-Stokes equa- 
tions in such a way that all persisting eddies are resolved. If the Reynolds number Re of the flow becomes very large, small flow structure will develop. Using the Kolmogorov law (see [2]) one can estimate the number of mesh cells needed to capture all persisting eddies. Such calculations show that DNS is possible on present day computers only to very limited values of the Reynolds number. Another difficulty for a DNS is that the boundary must have the precision which is required by the smallest scales of the flow. For real applications like geophysical flows it seems to be impossible to obtain precise boundary data. The impossibility of prescribing precise initial and boundary conditions causes the resulting flow to have random character. Thus DNS is not feasible for most turbulent flows.

1.2. Filtering technique. We will assume that every filter function $\varphi_{\delta}$ satisfies the following conditions:

1. $\varphi_{\delta}$ is a nonnegative $C^{\infty}$-function of compact support contained in $\Omega$,

2. the filter width $\delta$ is a positive function that depends on $x$ and $\Omega$, namely $\delta(x)=\operatorname{dist}(x, \partial \Omega)$,

3. the support of $\varphi_{\delta}$ shrinks to a one-point set near the boundary,

4. the mass of the filter remains 1 .

To be more precise, let $\varphi \in C_{0}^{\infty}(\Omega)$ be nonnegative such that $\int_{\Omega} \varphi(y) d y=1$, $\varphi(x)=\varphi(-x)$. Then we define the filter $\varphi_{\delta(x)}$ by

$$
\varphi_{\delta(x)}(y)=\frac{1}{\delta(x)^{3}} \varphi\left(\frac{y}{\delta(x)}\right)
$$

and the filtering is defined by

$$
\tilde{v}(x)=\int_{\Omega} v(y) \varphi_{\delta(x)}(x-y) d y .
$$

For brevity, we will usually write $\varphi_{\delta}$ instead of $\varphi_{\delta(x)}$ but throughout this paper we assume that $\delta$ depends on $x$.

For applications of filters with nonuniform filter width in numerical analysis we refer to [14.

Remark. Conditions 1-4 above imply that the filter functions tend to Dirac $\delta$-distributions on the boundary in the sense that

$$
\lim _{\delta(x) \rightarrow 0} \int_{\Omega} v(y) \varphi_{\delta(x)}(x-y) d y=v(x) .
$$

1.3. Function spaces and notation. This section sets the notation used in the paper and describes the function spaces employed. The notation used is standard for the most part. The $L^{p}(\Omega)$ norm is explicitly denoted as $\|\cdot\|_{L^{p}(\Omega)}$. The Sobolev spaces $W^{k, p}(\Omega)$ are defined in the usual way [1]. The associated norm is denoted by $\|\cdot\|_{W^{k, p}(\Omega)}$. The space $L^{2}(\Omega)$ is a Hilbert 
space with inner product and norm

$$
(u, v):=\int_{\Omega} u \cdot v d x, \quad\|u\|_{L^{2}}:=(u, u)^{1 / 2} \quad \text { for } u, v \in L^{2}(\Omega) .
$$

$L_{0}^{2}(\Omega)$ denotes the space of functions in $L^{2}(\Omega)$ with mean zero,

$$
L_{0}^{2}(\Omega):=\left\{u \in L^{2}(\Omega): \int_{\Omega} u d x=0\right\} .
$$

For simplicity, the domain $\Omega$ is sometimes omitted (e.g., $L^{2}(\Omega)=L^{2}$ ) and function spaces for vector-valued functions are denoted in the same way as function spaces for scalar functions.

Now we introduce some nonstandard function spaces used in the paper. By $\mathcal{D}(\Omega)$ we denote the space of all $C^{\infty}$-functions with compact support in $\Omega$ with values in $\mathbb{R}^{d}(d=2$ or 3$)$. Let us define

$$
\begin{aligned}
\mathcal{V} & :=\{u: u \in \mathcal{D}(\Omega), \operatorname{div} u=0\}, \\
V & :=\text { completion of } \mathcal{V} \text { in the norm }\|u\|_{V}=\left(\int_{\Omega}|D u|^{3} d x\right)^{1 / 3}, \\
V^{\prime} & :=\text { the dual space of } V .
\end{aligned}
$$

The duality pairing between $V$ and $V^{\prime}$ will be denoted by $\langle\cdot, \cdot\rangle$.

1.4. Weak solutions. In $[8]$ the following existence theorem was proved (which we will also use as the definition of a weak solution to (1.1)):

THEOREM 1.1. Given $f \in V^{\prime}$ there exists a weak solution to the stationary problem (1.1), i.e. the equation

$$
\int_{\Omega}(v \cdot \nabla v \cdot \phi+A(y, D v) \cdot D \phi+\nu \nabla v \cdot \nabla \phi-f \cdot \phi) d x=0
$$

is satisfied for all $\phi \in V$.

It will be useful to introduce the trilinear form

$$
b(u, v, w):=\int_{\Omega}(u \cdot \nabla v \cdot w) d x
$$

defined for $u, v, w \in V$.

2. Young measure tools. For the convenience of the reader we collect below all the necessary and non-standard tools concerning Young measures used in the proof of Theorem 5.1 below. For more details, definitions and proofs, we refer the reader to Chapter 3 in [10].

Lemma 2.1. Suppose that a sequence of maps $z^{j}: \Omega \rightarrow \mathbb{R}^{d}$ generates a Young measure $\nu$. Let $F: \Omega \times \mathbb{R}^{d} \rightarrow \mathbb{R}$ be a Carathéodory function (i.e. measurable in the first argument and continuous in the second). Moreover 
assume that the sequence of the negative parts $F^{-}\left(x, z^{j}(x)\right)$ is weakly relatively compact in $L^{1}(\Omega)$. Then

$$
\liminf _{j \rightarrow \infty} \int_{\Omega} F\left(x, z^{j}(x)\right) d x \geq \iint_{\Omega} F(x, \lambda) d \nu_{x}(\lambda) d x .
$$

If, in addition, the sequence of functions $x \mapsto|F|\left(x, z^{j}(x)\right)$ is weakly relatively compact in $L^{1}(\Omega)$, then

$$
F\left(\cdot, z^{j}(\cdot)\right) \rightarrow \int_{\mathbb{R}^{d}} F(x, \lambda) d \nu_{x}(\lambda) \quad \text { in } L^{1}(\Omega) .
$$

REMARK. The second part of the above theorem can be easily extended to vector-valued functions $F$.

LEMMA 2.2. Let $u^{j}: \Omega \rightarrow \mathbb{R}^{d}$ and $v^{j}: \Omega \rightarrow \mathbb{R}^{d}$ be measurable and suppose that $u^{j} \rightarrow u$ a.e. while $v^{j}$ generates a Young measure $\nu$. Then the sequence of pairs $\left(u^{j}, v^{j}\right): \Omega \rightarrow \mathbb{R}^{d+d^{6}}$ generates the Young measures $x \mapsto$ $\delta_{u(x)} \otimes \nu_{x}$

LEMMA 2.3. Suppose that a sequence $z^{j}$ of measurable functions from $\Omega$ to $\mathbb{R}^{d}$ generates a Young measure $\nu: \Omega \rightarrow \mathcal{M}\left(\mathbb{R}^{d}\right)$. Then

$$
z^{j} \rightarrow z \text { in measure if and only if } \nu_{x}=\delta_{z(x)} \text { a.e. }
$$

3. Discretization. Since we are particularly interested in finite element approximations let us introduce some typical assumptions that are used in this type of approximation.

For simplicity we assume that $\Omega$ is a polygonal domain and a regular and admissible finite-element triangulation $\mathcal{K}^{h}$ is given (see Definition 5.1 in [3]), where $h>0$ is a discretization parameter that tends to zero.

Restriction to admissible and regular triangulations is not crucial. It guarantees the existence of finite-dimensional spaces which we will use for finite-element approximation and it will also allow us to omit some technical difficulties.

Next we define two finite-dimensional spaces

$$
X^{h} \subset W_{0}^{1,3} \text { and } P^{h} \subset L_{0}^{2}
$$

such that $\bigcup_{h \downarrow 0} X^{h}$ is dense in $W_{0}^{1,3}$ and $\bigcup_{h \downarrow 0} P^{h}$ is dense in $L_{0}^{2}$. We will use these spaces to seek for an approximate solution (velocity and pressure respectively).

We will assume that $X_{K}$, the set of restrictions of the functions in $X_{h}$ to a single simplex $K$ in the triangulation, is a subset of the set containing all the polynomials defined on $K$ of degree at most $l$ ( $l$ is a fixed integer). Recently a very popular example of a finite-element function space satisfying the above conditions is the space consisting of Taylor-Hood elements (see 
[4] for details). Other examples of finite-dimensional function spaces defined on regular and admissible triangulations can be found in [3] and [4].

Let us also define a subspace $V^{h}$ of $X^{h}$ by

$$
V^{h}:=\left\{v^{h} \in X^{h}:\left(q^{h}, \operatorname{div} v^{h}\right)=0, \forall q^{h} \in P^{h}\right\}
$$

with the corresponding norm

$$
\|u\|_{V^{h}}:=\|\nabla u\|_{L^{3}} .
$$

We assume that $V^{h}$ is nonempty. Working in this subspace will allow us to simplify our approximation problem. Note that most often $V^{h} \not \subset V$. Thus, we need an extension of the trilinear form $b(u, v, w)$ of the following form. For $u, v, w \in W^{1,3}$,

$$
b(u, v, w):=\frac{1}{2} \int_{\Omega}(u \cdot \nabla v \cdot w-u \cdot \nabla w \cdot v) d x .
$$

By direct calculation, we can see that if $u, v, w \in V$, then (3.8) gives

$$
b(u, v, w):=\int_{\Omega}(u \cdot \nabla v \cdot w) d x,
$$

which coincides with the original definition (1.5). Hence the extension is well defined. The extension above enables us to pursue the discussion in the finite-dimensional subspace $V^{h}$ without losing some symmetry properties.

Now we approximate (1.1) by the following discrete problem:

$(\mathrm{LPh})$ Find a pair $\left(u^{h}, p^{h}\right) \in X^{h} \times P^{h}$ such that

$$
\begin{aligned}
\left(A\left(y^{h}, D u^{h}\right), D w^{h}\right)+b\left(u^{h}, u^{h}, w^{h}\right)+\nu\left(\nabla u^{h}, \nabla w^{h}\right)-\left(p^{h}, \operatorname{div} w^{h}\right) & =\left(f, w^{h}\right), \\
\left(q^{h}, \operatorname{div} u^{h}\right) & =0,
\end{aligned}
$$

for all $w^{h} \in X^{h}$, and $q^{h} \in P^{h}$.

Next we formulate another problem in $V^{h}$ associated with (LPh), which (without loss of generality) will simplify our considerations:

(LQh) Find $u^{h} \in V^{h}$ such that for all $w^{h} \in V^{h}$ :

$$
\left(A\left(y^{h}, D u^{h}\right), D w^{h}\right)+b\left(u^{h}, u^{h}, w^{h}\right)+\nu\left(\nabla u^{h}, \nabla w^{h}\right)=\left(f, w^{h}\right) .
$$

In our further discussion we will concentrate only on the (LQh) problem. We assume that the so-called inf-sup condition is satisfied.

Definition 3.1. The pair of finite-dimensional subspaces $X^{h}$ and $P^{h}$ satisfies the inf-sup condition if there exists a constant $\beta>0$, independent of $h$, such that

$$
\inf _{q \in P^{h}, q \neq 0} \sup _{v \in X^{h}, v \neq 0} \frac{(\operatorname{div} v, q)}{\|q\|_{L^{2}}\|\nabla v\|_{L^{2}}} \geq \beta .
$$


The inf-sup condition guarantees that for a given solution $u^{h}$ to (LQh), there exists $p^{h}$ such that $\left(u^{h}, p^{h}\right)$ satisfies $(\mathrm{LPh})$. See [4] for details.

4. Existence of the solution to the discrete problem. The following result is a consequence of the Brouwer fixed point theorem (see e.g. [6]).

THEOREM 4.1. For every fixed $h$ problem (LQh) has at least one solution in $V^{h}$. Moreover, if $u^{h}$ is a solution, then

$$
\frac{k}{2}\left\|u^{h}\right\|_{V^{h}}^{3}+\nu\left\|\nabla u^{h}\right\|_{L^{2}}^{2} \leq K\|f\|_{\left(V^{h}\right)^{\prime}}
$$

for some positive constants $K$ and $k$.

Proof. First we will prove the existence. Let $P: V^{h} \rightarrow V^{h}$ be defined by

$$
\begin{aligned}
{[P(v), w]=} & \int_{\Omega} c(y)|D v| D v \cdot D w d x \\
& +\nu(\nabla v, \nabla w)+b(v, v, w)-\langle f, w\rangle \quad \forall v, w \in V^{h} .
\end{aligned}
$$

Taking $w=v$ in (4.13), using the properties of $c$ and the Korn inequality, we obtain

Thus

$$
\begin{aligned}
{[P(v), v] } & =\nu\|\nabla v\|_{L^{2}(\Omega)}^{2}+\int_{\Omega} c(y)|D v|^{3} d x-\langle f, v\rangle \\
& \geq \nu\|\nabla v\|_{L^{2}(\Omega)}^{2}+\alpha \int_{\Omega}|D v|^{3} d x-\|f\|_{\left(V^{h}\right)^{\prime}}\|v\|_{V^{h}} \\
& \geq \nu\|\nabla v\|_{L^{2}(\Omega)}^{2}+k\|v\|_{W^{1,3}(\Omega)}^{3}-\|f\|_{\left(V^{h}\right)^{\prime}}\|v\|_{V^{h}} \\
& \geq \nu\|\nabla v\|_{L^{2}(\Omega)}^{2}+\|v\|_{V^{h}}\left(k\|v\|_{V^{h}}^{2}-\|f\|_{\left(V^{h}\right)^{\prime}}\right) .
\end{aligned}
$$

$$
[P(v), v]>0 \quad \text { for }\|v\|_{V^{h}} \leq K
$$

where $K>\left(\|f\|_{\left(V^{h}\right)^{\prime}} / k\right)^{1 / 2}$. Therefore there exists a $v^{h}$ such that $P\left(v^{h}\right)=0$.

Now we can prove the energy estimate $(4.12)$. Without loss of generality we can assume that $\operatorname{dim}\left(V^{h}\right)=n$. Let $\left\{\omega_{i}^{h}\right\}_{i=1}^{n}$ be a basis of $V^{h}$. Then the solution to (LQh) is defined by

$$
u^{h}(x)=\sum_{i=1}^{n} \lambda_{i}^{h} \omega_{i}^{h}, \quad \lambda_{i}^{h} \in \mathbb{R},
$$

where $v^{h} \in V^{h}=\operatorname{span}\left\{\omega_{1}^{h}, \ldots, \omega_{n}^{h}\right\}$ solves the system of equations

$$
\left(A\left(y^{h}, D u^{h}\right), D \omega_{i}^{h}\right)+b\left(u^{h}, u^{h}, \omega_{i}^{h}\right)+\nu\left(\nabla u^{h}, \nabla \omega_{i}^{h}\right)=\left(f, \omega_{i}^{h}\right)
$$

for $i=1, \ldots, n$. Multiplying 4.15 by $\lambda_{h}^{h}$ and summing over $i$ yields

$$
\left(A\left(y^{h}, D u^{h}\right), D u^{h}\right)+\nu\left(\nabla u^{h}, \nabla u^{h}\right)=\left(f, u^{h}\right) .
$$

This immediately leads to the estimate 4.12. 
5. Convergence theorem. Throughout this section we will not use subsequence indices even if a certain property, particularly convergence, holds only for a subsequence.

TheOREM 5.1. Assume $v$ is a weak solution to (1.1) and $u^{h}$ solves (LQh). Then there exists a subsequence of $u^{h}$ such that

$$
\lim _{h \rightarrow 0}\left\|v-u^{h}\right\|_{V^{h}}=0 .
$$

Before proving Theorem 5.1 note that $y \in \mathbb{R}^{d} \times \mathbb{S}^{d} \times \mathbb{S}^{d} \times \mathbb{S}^{d}$ and $D v \in \mathbb{S}^{d}$ where $\mathbb{S}^{d}$ denotes the set of symmetric matrices in $\mathbb{R}^{d \times d}$. Noting that $\mathbb{S}^{d} \subset$ $\mathbb{R}^{d^{2}}$ we see that $A$ is a mapping from $\mathbb{R}^{2 d+4 d^{2}}$ to $\mathbb{R}^{d^{2}}$. For simplicity we set $m=2 d+4 d^{2}$ and $n=d^{2}$. Now we can turn to the proof of Theorem 5.1.

Proof. Due to the energy estimate 4.12 it follows that there exists $u \in V$ such that

$$
u^{h} \rightarrow u \quad \text { in } V,
$$

which implies that there exist $\bar{A}$ and $\chi$ such that

$$
\begin{aligned}
u^{h} & \rightarrow u & & \text { in } L^{q}(\Omega) \text { for } 1 \leq q<\infty, \\
b\left(u^{h}, u^{h}, \phi\right) & \rightarrow b(u, u, \phi) & & \text { for all } \phi \in W^{1,3}, \\
A\left(y^{h}, D u^{h}\right) & \rightarrow \bar{A} & & \text { in } L^{3 / 2}(\Omega), \\
\left|D u^{h}\right| D u^{h} & \rightarrow \chi & & \text { in } L^{3 / 2}(\Omega) .
\end{aligned}
$$

Using the properties of convolution one can show (see Appendix for details) that

$$
y^{h} \rightarrow \bar{y} \quad \text { in } L^{\infty}(\Omega)
$$

where $y^{h}=\left(\widetilde{u^{h}}, \widetilde{D u^{h}}, \widetilde{u^{h} u^{h}}, \mid \widetilde{D u^{h} \mid D} u^{h}\right)$ and $\bar{y}=(\widetilde{u}, D \widetilde{u}, \widetilde{u u}, \widetilde{\chi})$. Now, letting $h \searrow 0$ in (LQh) we obtain

$$
b(u, u, \phi)+\int_{\Omega} \bar{A} \cdot D \phi d x+\nu(\nabla u, \nabla \phi)=\langle f, \phi\rangle .
$$

We claim that $u$ solves 1.1$)$. To prove it we will show that $\bar{A}=A(y, D u)$ and

$$
\lim _{h \rightarrow 0}\left\|u-u^{h}\right\|_{V^{h}}=0 .
$$

The function $A\left(y^{h}, D u^{h}\right) \cdot D u^{h}$ satisfies the inequality

$$
A\left(y^{h}, D u^{h}\right) \cdot D u^{h} \geq \alpha\left|D u^{h}\right|^{3},
$$

which ensures that the negative part of this function is equal to zero. Thus the sequence $A\left(y^{h}, D u^{h}\right) \cdot D u^{h}$ is certainly weakly relatively compact in 
$L^{1}(\Omega)$. Applying Lemma 2.1 we conclude that

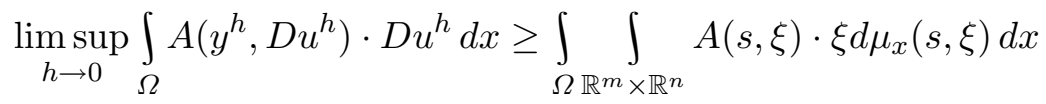

where $\mu_{x}$ is the Young measure generated by the sequence $\left(y^{h}, D u^{h}\right)$. However, according to Lemma 2.2 we are able to characterize this Young measure more precisely. Since

$$
y^{h} \rightarrow \bar{y} \quad \text { in } L^{\infty}(\Omega)
$$

and $D u^{h}$ generates the Young measure $\nu_{x}$, the Young measure $\mu_{x}$ generated by this pair satisfies $\mu_{x}=\delta_{\bar{y}(x)} \otimes \nu_{x}$. Therefore we can integrate

$$
\int_{\Omega \mathbb{R}^{m} \times \mathbb{R}^{n}} \int_{\Omega} A(s, \xi) \cdot \xi d \mu_{x}(s, \xi) d x=\iint_{\Omega \mathbb{R}^{n}} A(\bar{y}(x), \xi) \cdot \xi d \nu_{x}(\xi) d x .
$$

In the same way we obtain

$$
\int_{\Omega \mathbb{R}^{m} \times \mathbb{R}^{n}} \int_{\Omega} A(s, \xi) d \mu_{x}(s, \xi) d x=\iint_{\Omega \mathbb{R}^{n}} A(\bar{y}(x), \xi) d \nu_{x}(\xi) d x .
$$

Since the sequence $\left|A\left(y^{h}, D u^{h}\right)\right|$ (due to the energy estimate 4.12) is bounded in $L^{3 / 2}(\Omega)$, it is weakly relatively compact in $L^{1}(\Omega)$ and it follows that

$$
A\left(y^{h}, D u^{h}\right) \rightarrow \bar{A} \quad \text { in } L^{3 / 2}(\Omega)
$$

where

$$
\bar{A}(x)=\int_{\mathbb{R}^{n} \times \mathbb{R}^{m}} A(s, \xi) d \mu_{x}(s, \xi) .
$$

The approximate equation yields

$$
\int_{\Omega} A\left(y^{h}, D u^{h}\right) \cdot D u^{h} d x=-\nu\left\|D u^{h}\right\|_{L^{2}}^{2}+\int_{\Omega} f u^{h} d x,
$$

and thus due to the lower semicontinuity of the norm,

$$
\limsup _{h \rightarrow 0} \int_{\Omega} A\left(y^{h}, D u^{h}\right) \cdot D u^{h} d x \leq-\nu\|D u\|_{L^{2}}^{2}+\int_{\Omega} f u d x .
$$

Thus, combining (5.22)-5.27) we obtain

$$
\begin{aligned}
\iint_{\Omega \mathbb{R}^{n}} A(\bar{y}(x), \xi) d \nu_{x}(\xi) \cdot D u d x & \stackrel{5.24}{=} \int_{\Omega \mathbb{R}^{n} \times \mathbb{R}^{m}} \int_{(s, \xi) d \mu_{x}(s, \xi) \cdot D u d x} A(x) \\
& \int_{\Omega} \bar{A} \cdot D u d x \\
& \stackrel{5.27}{\geq} \limsup _{h \rightarrow 0} \int_{\Omega} A\left(y^{h}, D u^{h}\right) \cdot D u^{h} d x
\end{aligned}
$$




$$
\begin{aligned}
& \stackrel{5.22}{\geq} \int_{\Omega} \int_{\mathbb{R}^{m} \times \mathbb{R}^{n}} A(s, \xi) \cdot \xi d \mu_{x}(s, \xi) d x \\
& \stackrel{5.23}{=} \int_{\Omega} A(\bar{y}, \xi) \cdot \xi d \nu_{x}(\xi) d x,
\end{aligned}
$$

and so

$$
\iint_{\Omega} A(\bar{y}(x), \xi) d \nu_{x}(\xi) \cdot D u d x \geq \int_{\Omega} \int_{\mathbb{R}^{n}} A(\bar{y}, \xi) \cdot \xi d \nu_{x}(\xi) d x .
$$

From the monotonicity of $A$ in the last variable we can deduce that

$$
\iint_{\Omega} H(\xi) d \nu_{x}(\xi) d x \geq 0
$$

where $H$ is defined by

$$
H(\xi):=[A(\bar{y}, \xi)-A(\bar{y}, D u)] \cdot[\xi-D u] .
$$

Simple calculations imply that

$$
\begin{aligned}
\iint_{\Omega} H(\xi) d \nu_{x}(\xi) d x= & \int_{\Omega} \int_{\mathbb{R}^{n}} A(\bar{y}, \xi) \cdot \xi d \nu_{x}(\xi) d x \\
& -\iint_{\Omega} A(\bar{y}, \xi) d \nu_{x}(\xi) \cdot D u d x,
\end{aligned}
$$

which together with $(5.28)$ ensures that

$$
\iint_{\Omega} H(\xi) d \nu_{x}(\xi) d x \leq 0 .
$$

Then 5.29 and 5.32 imply that $\int_{\mathbb{R}^{n}} H(\xi) d \nu_{x}(\xi)=0$ for a.a. $x \in \Omega$. Moreover, since $\nu_{x} \geq 0$ is a probability measure and $H(\xi)$ is strictly monotone, we have

$$
\operatorname{supp}\left\{\nu_{x}\right\} \stackrel{\text { a.e. }}{=}\{D u(x)\} \text {. }
$$

Note that the single point on the right-hand side set is located a.e. at the point $D u(x)$, where $D u$ is the weak limit of the sequence $\left(D u^{h}\right)$. Hence we conclude that $\nu_{x}=\delta_{D u(x)}$ a.e. A direct application of Lemma 2.3 shows that

$$
D u^{h} \rightarrow D u \quad \text { in measure. }
$$

Hence there exists a subsequence of $\left(D u^{h}\right)$ such that $D u^{h} \rightarrow D u$ a.e. Using the fact that $\nu_{x}=\delta_{D u(x)}$ together with Lemma 2.1 and (5.27) yields

$$
\begin{aligned}
\limsup _{h \rightarrow 0} \int_{\Omega} A\left(y^{h}, D u^{h}\right) \cdot D u^{h} d x & \leq \int_{\Omega} A(\bar{y}(x), D u) \cdot D u d x \\
& \leq \liminf _{h \rightarrow 0} \int_{\Omega} A\left(y^{h}, D u^{h}\right) \cdot D u^{h} d x .
\end{aligned}
$$


Hence we can set $g^{h}=A\left(y^{h}, D u^{h}\right) \cdot D u^{h}, g=A(\bar{y}, D u) \cdot D u$ and claim that

$$
g^{h} \geq 0, \quad g \in L^{1}(\Omega), \quad \int_{\Omega} g^{h} d x \rightarrow \int_{\Omega} g, \quad g^{h} \rightarrow g \quad \text { a.e. in } \Omega .
$$

Noticing that

$$
\int_{\Omega}\left|g^{h}-g\right| d x=\int_{\Omega}\left(g^{h}-g\right) d x+2 \int_{\left\{x: g^{h} \leq g\right\}} \max \left\{g-g^{h}, 0\right\} d x
$$

we conclude by Lebesgue's Dominated Convergence Theorem that

$$
A\left(y^{h}, D u^{h}\right) \cdot D u^{h} \rightarrow A(\bar{y}, D u) \cdot D u \quad \text { in } L^{1}(\Omega) .
$$

Thus by Vitali's Theorem, $A\left(y^{h}, D u^{h}\right) \cdot D u^{h}$ is uniformly integrable. Due to the coercivity condition, also the sequence $\left|D u^{h}\right|^{3}$ is uniformly integrable. Using again Vitali's Theorem yields

$$
D u^{h} \rightarrow D u \quad \text { in } L^{3}(\Omega) .
$$

We still have to prove that $u$ solves (1.1). First, recall that

$$
D u^{h} \stackrel{\text { a.e. }}{\longrightarrow} D u
$$

(the convergence may hold only for a subsequence). Hence

$$
\left|D u^{h}\right| D u^{h} \stackrel{\text { a.e. }}{\longrightarrow}|D u| D u,
$$

which together with (5.19) implies that $\chi=|D u| D u$ a.e. in $\Omega$. Thus $y=\bar{y}$ a.e. in $\Omega$ and also

$$
c\left(y^{h}\right)\left|D u^{h}\right| D u^{h} \rightarrow c(y)|D u| D u \quad \text { a.e. in } \Omega .
$$

The term $c\left(y^{h}\right)\left|D u^{h}\right| D u^{h}$ is bounded in $L^{3 / 2}(\Omega)$. Thus we can conclude that

$$
c\left(y^{h}\right)\left|D u^{h}\right| D u^{h} \rightarrow c(y)|D u| D u \quad \text { in } L^{3 / 2}(\Omega),
$$

which completes the proof.

6. Appendix. In the proof of the convergence theorem we used the fact that

$$
y^{h} \rightarrow \bar{y} \quad \text { in } L^{\infty}(\Omega) .
$$

In [12] and [13] analogous facts were proved for periodic boundary conditions and for the time-dependent model respectively. In this paper we consider the stationary case, we do not limit ourselves to periodic boundary conditions and we also use a different filtering technique. For completeness we will prove the convergence (6.35) although the reasoning is almost identical to those presented in [12] and [13]. Let us start with the following lemma (we use the notation introduced in the previous sections):

Lemma 6.1. Let the sequence $\left(u^{h}\right)$ converge weakly to $v$ in $V$ as $h \rightarrow 0$. Then there exists $\chi \in L^{3 / 2}(\Omega)$ such that for $h \rightarrow 0$ the following sequences 
converge almost everywhere in $\Omega$ :

$$
\widetilde{u^{h}} \rightarrow \widetilde{u}, \quad \widetilde{u^{h} u^{h}} \rightarrow \widetilde{u u}, \quad \widetilde{D u^{h}} \rightarrow \widetilde{D u}
$$

We can extract a further subsequence of $\left(u^{h}\right)$ such that

$$
\mid \widetilde{D u^{h} \mid D} u^{h} \rightarrow \widetilde{\chi} \quad \text { a.e. in } \Omega \text {. }
$$

Proof. Since the sequence $u^{h}$ is bounded in $V$, we also have $D u^{h} \rightarrow D u$ in $L^{3}(\Omega)$ and $u^{h} \rightarrow u^{h}$ in $L^{3}(\Omega)$, so that

$$
\int_{\Omega} u^{h} \phi d y \rightarrow \int_{\Omega} u \phi d y \quad \forall \phi \in L^{3 / 2}(\Omega) .
$$

We choose as test function $\phi(y)=\varphi_{\delta(x)}(x-y)$ with parameter $x \in \Omega$, where $\varphi_{\delta(x)}$ is the filter. As the filter is a $C^{\infty}$-function of compact support, $\phi(y)$ is in $L^{3 / 2}(\Omega)$ unless the support is equal to one point and the filter becomes a Dirac $\delta$-distribution. But these are the points from the boundary and therefore

$$
\int_{\Omega} u^{h}(y) \varphi_{\delta(x)}(x-y) d y \rightarrow \int_{\Omega} u(y) \varphi_{\delta(x)}(x-y) d y \quad \text { for all } x \in \Omega,
$$

which is equivalent to

$$
\widetilde{u^{h}} \rightarrow \tilde{u} \quad \text { a.e. in } \Omega \text {. }
$$

In the same way from the information on the symmetric part of the gradients we conclude that

$$
\widetilde{D u^{h}} \rightarrow \widetilde{D u} \quad \text { a.e. in } \Omega \text {. }
$$

To get the limit of the sequence $\widetilde{u^{h} u^{h}}$ we deduce from the strong convergence of the sequence $u^{h}$ in $L^{2}(\Omega)$ also the strong convergence of $u^{h} u^{h}$ to $u u$ in $L^{1}(\Omega)$. Of course strong convergence implies weak convergence; thus analogous arguments yield

$$
\widetilde{u^{h} u^{h}} \rightarrow \widetilde{u u} \quad \text { a.e. in } \Omega \text {. }
$$

Since $D u^{h}$ is bounded in $L^{3}(\Omega)$, we conclude that $\left|D u^{h}\right| D u^{h}$ is bounded in $L^{3 / 2}(\Omega)$; thus, for a subsequence,

$$
\left|D u^{h}\right| D u^{h} \rightarrow \chi \quad \text { in } L^{3 / 2}(\Omega) .
$$

Then again for the filtered terms we have

$$
\mid \widetilde{D u^{h} \mid D u^{h}} \rightarrow \tilde{\chi} \quad \text { a.e. in } \Omega \text {. }
$$

Now the convergence 6.35) follows immediately.

Acknowledgements. The author would like to thank Dr. Agnieszka Świerczewska-Gwiazda for her careful reading of this paper and numerous fruitful discussions. 
This work was written during the author's stay at Nečas Center for Mathematical Modeling in Prague.

\section{References}

[1] R. A. Adams and J. J. F. Fournier, Sobolev Spaces, 2nd ed., Pure Appl. Math. 14, Academic Press, 2003.

[2] L. C. Berselli, T. Iliescu, and W. J. Layton, Mathematics of Large Eddy Simulation of Turbulent Flows, Springer, 2006.

[3] D. Braess, Finite Elements. Theory, Fast Solvers and Applications in Solid Mechanics, Cambridge Univ. Press, 2007.

[4] F. Brezzi and M. Fortin, Mixed and Hybrid Finite Element Methods, Springer Ser. Comput. Math. 15, Springer, 1991.

[5] Q. Du and M. D. Gunzburger, Finite-element approximations of a Ladyzhenskaya model for stationary incompressible viscous flow, SIAM J. Numer. Anal. 27 (1990), $1-19$.

[6] L. C. Evans, Partial Differential Equations, Grad. Stud. Math. 19, Amer. Math. Soc., 1998.

[7] M. Germano, U. Piomelli, P. Moin, and W. H. Cabot, A dynamic subgrid-scale eddy viscosity model, Phys. Fluids A 3 (1991), 1760-1765.

[8] P. Gwiazda and A. Świerczewska, Large eddy simulation turbulence model with Young measures, Appl. Math. Lett. 18 (2005), 923-929.

[9] V. John, Large Eddy Simulation of Turbulent Incompressible Flows. Analytical and Numerical Results for a Class of LES Models, Lecture Notes in Comput. Sci. Engrg. 34, Springer, 2004.

[10] S. Müller, Variational models for microstructure and phase transitions, in: Calculus of Variations and Geometric Evolution Problems (Cetraro, 1996), Lecture Notes in Math. 1713, Springer, 1999, 85-210.

[11] P. Sagaut, Large Eddy Simulation for Incompressible Flows. An Introduction, Springer, 2001.

[12] A. Świerczewska, Large eddy simulation. Existence of stationary solutions to a dynamical model, Z. Angew. Math. Mech. 85 (2005), 593-604.

[13] -, A dynamical approach to large eddy simulation of turbulent flows: existence of weak solutions, Math. Methods Appl. Sci. 29 (2006), 99-121.

[14] H. van der Ven, A family of large eddy simulation (les) filters with nonuniform filter widths, Phys. Fluids 7 (1995), 1171-1172.

Andrzej Warzyński

School of Computing

University of Leeds

Leeds, LS2 9JT, UK

E-mail: scaw@leeds.ac.uk

Received on 19.2.2009;

revised version on 29.6.2009 
